\title{
Frecuencia de alexitimia y factores relacionados en pacientes con fibromialgia tratados en un hospital público de Lima, Perú.
}

Frequency and related factors of alexithymia in patients with fibromyalgia treated at a public hospital in Lima, Peru.

Kim Leslie Nestares Luna 1,a, Anapaula Olivera García ${ }^{1, a}$, Roberto Huamanchumo Guzmán ${ }^{1,2, b}$, Jorge Martín Arévalo Flores ${ }^{1,2, c}$

\section{RESUMEN}

Objetivo: Determinar la frecuencia de alexitimia y factores relacionados en pacientes con fibromialgia, y la relación entre alexitimia y capacidad funcional, intensidad de dolor, depresión y variables generales (edad, sexo, tiempo de enfermedad, grado de instrucción y años de estudio) en fibromialgia. Material y métodos: Se realizó un estudio descriptivo transversal en pacientes con fibromialgia del Servicio de Reumatología de un hospital público de Lima, Perú. Se aplicó una ficha sociodemográfica, la TAS-20, el MD-HAQ y la CES-D. Resultados: De los 126 pacientes con fibromialgia, 60 (47,62 \%) mostraron alexitimia, 24 de ellos presentaron depresión (40\%) y 39, discapacidad funcional (65\%). En el análisis bivariado la alexitimia se relacionó significativamente con mayor comorbilidad, menor grado de instrucción, mayor intensidad de dolor, mayor fatiga y más síntomas reportados. En el análisis multivariado, años de estudio ( $£ 11$ años) fue el único factor relacionado a alexitimia (TAS-20) ( $\mathrm{p}=0,010$, OR: 2,589 [1,249 - 5,365]). Conclusiones: Se demuestra una frecuencia considerable de alexitimia en pacientes con fibromialgia y, en el análisis multivariado, este hallazgo se relaciona significativamente con un número menor de años de estudio.

PALABRAS CLAVE: Alexitimia, fibromialgia, depresión.

\section{SUMMARY}

Objective: To determine the frequency of alexithymia and associated factors in patients with fibromyalgia, and the relationship between alexithymia with functional capacity, pain intensity, depression and general variables (age, sex, time of illness, degree of instruction, and years of study) in fibromyalgia. Material and methods: Descriptive cross-sectional study of patients with fibromyalgia from the Rheumatology service of a public hospital in Lima, Perú. A sociodemographic record, TAS-20, MD-HAQ and CES-D were applied. Results. Of the 126 patients with fibromyalgia, 60 had alexithymia (47.62\%); 24 alexithymic patients had depression (40\%), and 39 had functional disability $(65 \%)$. In the bivariate analysis, alexithymia was significantly related to greater comorbidity, less education, greater pain intensity, greater fatigue and more reported symptoms. In the multivariate analysis, years of study ( $£$ 11 years) were the only factor related to alexithymia (TAS-20) ( $p=0.010$, OR: 2.589 [1.249 - 5.365]). Conclusions: This study shows a considerable frequency of alexithymia in fibromyalgia patients and, in the multivariate analysis, the finding is significantly related to a lower number of years of study.

KEYWORDS: Alexithymia, fibromyalgia, depression.

Facultad de Medicina, Universidad Peruana Cayetano Heredia. Lima, Perú.

2 Hospital Nacional Cayetano Heredia. Lima, Perú.

a Bachiller en Medicina, ${ }^{b}$ Médico reumatólogo; ${ }^{\text {c }}$ Médico psiquiatra. 


\section{INTRODUCCIÓN}

La fibromialgia es una enfermedad reumatológica caracterizada por la presencia de dolor crónico músculo-esquelético que se presenta de manera difusa, no asociado a compromiso articular (1). Es una enfermedad que afecta al ser humano en el ámbito biológico, psicológico y social (2), su diagnóstico es de exclusión de otras enfermedades que puedan confundirse con fibromialgia.

El Colegio Americano de Reumatología (ACR) publicó en 1990 una lista de criterios diagnósticos para fibromialgia que fue actualizada en el 2010 y que ha sufrido una última modificación en el año 2016. Sin embargo, algunos estudios comparativos recomiendan utilizar los criterios del 2016 solo como herramienta de tamizaje, ya que posee una baja especificidad que oscila entre un $44-50 \%(1,3)$.

La prevalencia de esta enfermedad en países desarrollados es de 1-4\% (4). En un estudio realizado en España (EPISER 2000) se encontró una prevalencia de $2,4 \%$ a predominio de mujeres $(4,2 \%$ frente a $0,2 \%$ en hombres) con un pico de prevalencia entre los 40 y los 49 años (5). En el Perú, se realizó un estudio observacional retrospectivo donde se comparó la frecuencia de fibromialgia en el año 2009 y 2011 en un policlínico de Lima, en el cual se encontró un aumento en la frecuencia de la enfermedad de $2,33 \%$ a $3,44 \%$, siendo una diferencia significativa $(\mathrm{p}<0,001)(6)$.

Se ha visto en muchos estudios que la fibromialgia es más prevalente en mujeres que en hombres. En un estudio observacional a lo largo de 2 años en 3 centros de atención primaria en España se evaluó una muestra de 235 personas diagnosticadas con fibromialgia, obteniendo una frecuencia de población femenina de $97,8 \%$ con una edad media de 54,6 años (7). Otro estudio realizado en España reveló mayor frecuencia de fibromialgia en el sexo femenino. Además, al momento de realizar el análisis de varianza (ANOVA) se encontró que las personas con fibromialgia presentaron mayor índice psicopatológico liderando la somatización, obsesión-compulsión, depresión y ansiedad comparado a aquellas personas que no presentaban fibromialgia (8).

Como podemos observar, la fibromialgia es una enfermedad compleja que ha sido asociada a síntomas psicopatológicos que en la mayoría de los casos influyen en el origen y/o desarrollo de la enfermedad (9). Una de las variables psicopatológicas más estudiadas asociada a fibromialgia ha sido la depresión, la cual se ha encontrado en un alto porcentaje tanto en su forma moderada como severa (10). Otra característica psicopatológica altamente relacionada a la fibromialgia es la alexitimia. Se ha descrito la alexitimia como una "característica de la personalidad en la cual hay dificultad para identificar y verbalizar emociones" (11). La persona no tiene suficiente capacidad de identificar sentimientos debido a la interiorización de las emociones, que al procesarlos puede desenlazar, como medio de expresión, en síntomas físicos (10).

Se ha observado que la frecuencia varía en diferentes poblaciones. En Chile se reportó una frecuencia de alexitimia del $10 \%$ en la población fibromiálgica estudiada (12), Avilaet al., en Brasil, al estudiar una población de 40 personas, evidenciaron mayor presencia de alexitimia en los pacientes con fibromialgia, comparado al grupo control (13).Por otro lado, un estudio realizado en México se reportó una frecuencia de alexitimia del 64,5\% (14). Referente a los estudios europeos donde se han realizado más trabajos con respecto a la presencia de alexitimia, en un estudio en Turquía se encontró una frecuencia de alexitimia de $24,29 \%$ en pacientes con diagnóstico de fibromialgia lo cual fue significativamente mayor sobre los que no presentaron fibromialgia (15). Otro estudio que encontró una frecuencia similar a la anterior fue uno realizado en Italia en el año 2012 donde se reportó un $20 \%$ de alexitimia dentro de la población con fibromialgia (16).

Hay estudios que han evaluado la relación de alexitimia con otras variables en la población fibromiálgica, encontrando mayor presencia de depresión, severidad de fibromialgia y menor calidad de vida en términos de dolor, sueño, reacciones emocionales e interacciones sociales (15).

Martínez y col. encontraron correlación entre dolor y alexitimia, pero no observaron una relación con capacidad funcional (17), si bien el estudio anterior concluye que la presencia de alexitimia no es un predictor significativo de la experiencia del dolor; en el estudio de Gálvez y col. se evidencia que la alexitimia se relaciona negativamente con la atención y cognición en pacientes con fibromialgia, siendo esos dos últimos factores importantes al relacionarse con la gravedad del dolor (9). Horta-Bass et al., encontraron dentro de sus resultados mayor intensidad de dolor, ansiedad, depresión, percepción de discapacidad 
Frecuencia de alexitimia y factores relacionados en pacientes con fibromialgia tratados en un hospital público de Lima.

y menor calidad de vida en personas que padecen fibromialgia y alexitimia comparado a aquellas que solo tienen fibromialgia (14).

La alexitimia ha tomado importancia durante los últimos años, por lo que no se cuenta con muchos estudios. En el Perú no se han encontrado estudios que relacionen fibromialgia y alexitimia, lo cual genera interés de obtener información de dicha relación ya que una frecuencia considerable de alexitimia dentro de la población con fibromialgia podría cambiar la perspectiva del manejo de dichos pacientes.

Los objetivos de este estudio fueron determinar la frecuencia de alexitimia y sus factores asociados en pacientes con fibromialgia. Además, de hallar la relación entre alexitimia y capacidad funcional, intensidad de dolor, depresión, y variables generales (edad, sexo, tiempo de enfermedad, grado de instrucción y años de estudio) en pacientes con diagnóstico de fibromialgia.

\section{MATERIAL Y MÉTODOS}

El diseño del presente estudio fue descriptivo transversal en una población de pacientes con fibromialgia del servicio de reumatología del Hospital Cayetano Heredia $(\mathrm{HCH})$.

1. Criterios de inclusión

- Pacientes con diagnóstico de fibromialgia

- $\quad$ Rango de edad: 18 a 65 años

- Pacientes que firmen el consentimiento informado.

- Pacientes con grado de instrucción secundaria (incompleta o completa) o superior.

2. Criterios de exclusión

- Pacientes con comorbilidades autoinmunes.

- Pacientes con enfermedades metabólicas descompensadas.

- Pacientes con historia de consumo de alcohol y sustancias ilícitas.

- Pacientes con trastornos psicóticos.

- Pacientes con tratamiento psiquiátrico al momento de la evaluación.

- $\quad$ Pacientes con grado de instrucción primaria.

El tamaño muestral fue calculado bajo la fórmula donde se estima prevalencia: $\mathrm{N}=\left(\mathrm{z}^{\wedge} 2 \mathrm{pq}\right) / \mathrm{E}^{\wedge} 2$. Se consideró un $\mathrm{p}=20 \%$, según los resultados de un estudio previo en Italia (16), $\mathrm{q}=80 \%, \mathrm{E}= \pm 7 \%$. Se seleccionaron los pacientes con diagnóstico de fibromialgia del servicio de reumatología durante el mes de febrero 2020 que cumplieran con los criterios de selección y firmen el consentimiento informado. La muestra obtenida fue de 126 pacientes del servicio de reumatología del $\mathrm{HCH}$, diagnosticados con fibromialgia en base a los criterios del 2010 de la Sociedad Americana de Reumatología. Los participantes fueron evaluados con la Escala de Toronto para Alexitimia (TAS-20), la Escala para Depresión del Centro de Estudios Epidemiológico (CES-D) y el Cuestionario Multidimensional de Evaluación de la Salud (MD-HAQ) para la valoración de la capacidad funcional.

Las variables independientes fueron:

a. Alexitimia: Puntaje mayor o igual a 61 con el TAS20.

b. Depresión: Puntaje final mayor igual a 29 con el CES-D.

c. Grado de instrucción: Nivel secundaria o superior. Información obtenida de la ficha sociodemográfica.

d. Tiempo de enfermedad: Valor en años con respecto al tiempo de enfermedad con diagnóstico de fibromialgia. Información obtenida de la ficha sociodemográfica.

e. Sexo: Masculino o femenino. Información obtenida de la ficha sociodemográfica.

f. Edad: Valor expresado en años. Información obtenida de la ficha sociodemográfica.

Las variables dependientes:

a. Capacidad funcional: se considera discapacidad funcional a valores iguales o mayores a la mediana $(0,4)$ de la primera parte del MD-HAQ.

b. Intensidad de dolor: Se usará la escala visual análoga del dolor presente en la segunda parte del MD-HAQ. La cual puntúa una escala del 0 10 , siendo 10 la escala máxima de dolor.

c. Síntomas asociados: Cantidad de síntomas del 0 al 60. Se utiliza la tercera parte del MD-HAQ.

Se aplicaron los siguientes instrumentos de evaluación: Ficha sociodemográfica: Se obtuvo datos como la edad, género, tiempo de enfermedad, tratamiento, comorbilidades y año de instrucción.

Escala de Toronto para alexitimia (TAS20): Evalúa la presencia de síntomas asociados a la alexitimia. Esta escala examina tres factores principales: capacidad para identificar y describir sentimientos, capacidad para diferenciar sentimientos de sensaciones corporales, capacidad para simbolizar y preferencia en enfocar eventos externos. Se puntúa 
mediante una escala de 5 puntos para cada ítem, que en total son 20, por lo que el puntaje total puede oscilar entre 20 y 100 . Se considera alexitímico a un paciente con puntaje mayor o igual a 61 . Ha sido validada en Perú por Kendall, R. y Rodríguez, en el año 2003, con un coeficiente de correlación de Pearson: factor 1 $(0,747)$, factor $2(0,575)$, factor $3(0,311)$; y un alfa de Cronbach de $78 \%$ (18).

Cuestionario multidimensional de evaluación de la salud (MD-HAQ): instrumento usado para evaluar el estado funcional de los pacientes. Consiste en tres partes: 1) versión modificada del HAQ que evalúa aspectos psicológicos del paciente; 2) escalas visuales análogas de dolor, actividad global de la enfermedad y fatiga; 3) información de síntomas generales. El puntaje de la primera parte se obtiene sumando cada ítem (cada uno tiene puntuación del 0 al 3) y dividiendo el total entre 10. Cada escala análoga tiene una puntuación de $0-10$, siendo 10 la puntuación máxima para cada variable. Fue validada en el Perú el año 2005 en pacientes con artritis reumatoide en un hospital general (19). El cuestionario tiene viabilidad con un tiempo de llenado de aproximadamente 11,7 minutos, tiene una validez de criterio superior al $70 \%$ al correlacionarlo con la observación directa (19).

Escala para depresión (CES-D): escala usada para la detección de trastornos depresivos. Está compuesta por 20 ítems, cada uno de los ítems se puntúa de 0 a 3 , obteniendo en total una puntuación total que va de 0 a 60. Fue validado en el Perú por Ruiz-Grosso y col. en el año 2012, con un alfa de Cronbach de 0,93. El punto de corte utilizado fue $\geq 29$ (20).

Se obtuvo la aprobación del comité de ética tanto de la Universidad Peruana Cayetano Heredia como del Hospital Cayetano Heredia.

El reclutamiento de lospacientes se realizó los días que presentaban cita en consultorio de reumatología, posterior a la atención dada por el médico tratante. Se solicitó al paciente su consentimiento para ser parte del estudio y disposición para poder desarrollar las evaluaciones correspondientes (TAS-20, CES-D y MD-HAQ). Los investigadores principales revisaron los datos de los participantes con el fin de que se cumpla con los criterios de selección.

La información fue recopilada a una base de datos. Únicamente los investigadores tuvieron acceso a esta base de datos. Las fichas de recolección fueron archivadas y guardadas por los investigadores con el fin de mantener la confidencialidad de datos obtenidos de acuerdo a la declaración de Helsinki de la Asociación Médica Mundial (AMM) (21). La base de datos virtual es propiedad de los investigadores principales.

Se utilizó el programa STATA 16 para el proceso estadístico de la base de datos obtenida durante el estudio. Luego del análisis en búsqueda de cumplir los objetivos de la investigación, se utilizaron las siguientes técnicas estadísticas:

1. Técnicas estadísticas descriptivas:

- Para evaluar la normalidad de las variables, se utilizó la prueba de Shapiro-Wilk.

- Mediana (Me) + Rango intercuartil (RIC): para evaluar variables de distribución no normal.

- $\quad$ Media (x) + Desviación estándar (S): para evaluar variables de distribución normal.

- Proporciones: para evaluar variables cualitativas como el género.

- Tablas y gráficos

2. Técnicas estadísticas inferenciales:

Se usó la técnica no paramétrica "U" de Mann Whitney para comparar medianas si la distribución no era normal, y "t de Student" para comparar medias si la distribución era normal.

Se realizó la regresión logística múltiple para estudiar la asociación conjunta entre: capacidad funcional, intensidad de dolor, depresión y características generales con alexitimia, obteniéndose en primer lugar los OR crudos y luego los OR ajustados (para evaluar los factores confusores).

\section{RESULTADOS}

Se evaluaron 126 pacientes con diagnóstico de fibromialgia. La frecuencia de alexitimia fue de $47,62 \%$, siendo la media del puntaje del TAS 20 de la muestra 59,4 (DS=12,2). Principalmente, se encontró una población femenina en un $96,83 \%$. La frecuencia de depresión fue de $33,3 \%$. Un $62,7 \%$ de la población fibromiálgica presentó comorbilidades y tratamiento farmacológico. Dentro de las comorbilidades, en la población alexitímica, se encuentran principalmente: artrosis $(22,02 \%)$, hipertensión arterial (16,95\%), diabetes mellitus tipo $2(15,25 \%)$, osteoporosis $(11,86 \%)$ y gastritis $(10,17 \%)$; como se describe en la tabla 3. El 60,32\% de la muestra presentó discapacidad y el mismo número rigidez matutina. La mediana de la edad de la muestra fue de 53,5 años (rango intercuartil: 
Frecuencia de alexitimia y factores relacionados en pacientes con fibromialgia tratados en un hospital público de Lima.

46-60,25), la mediana del número de síntomas físicos reportados durante el último mes previo a la evaluación fue 13 (rango intercuartil: 7-22). La mediana del número de años de estudio fue 11 (rango intercuartil: 11-14). La mediana del tiempo de enfermedad expresada en años fue 13 (rango intercuartil: 1,25-5). Todo lo anteriormente mencionado se encuentra en la tabla 1, donde se hace una descripción general de la población estudiada.

Tabla 1. Descripción de la población

\begin{tabular}{ll}
\hline & Total \\
$\mathbf{n}(\mathbf{\%})$
\end{tabular}

En la tabla 2 se hace una descripción de la relación encontrada entre alexitimia y las diferentes variables estudiadas. Al momento de realizar el análisis bivariado se encontró una relación significativa entre la presencia de alexitimia y alguna comorbilidad $(\mathrm{p}=0,047, \mathrm{OR}=2,107$ [1,009-4,399]). Otro resultado significativo fue la relación entre alexitimia y un nivel de grado secundaria (incluyendo tanto personas con secundaria completa como incompleta). No se encontró relación significativa de alexitimia con depresión y discapacidad, obteniendo un $\mathrm{p}=0,13$ y $\mathrm{p}=0,30$, respectivamente.

La intensidad de dolor tuvo una relación significativa con la presencia de alexitimia $(\mathrm{p}=0,0244)$. También se encontró una relación significativa entre menos años de estudio y alexitimia $(\mathrm{p}=0,0016)$. El número de comorbilidades presentes en la población con fibromialgia fue mayor en aquellos pacientes que presentaron alexitimia $(p=0,0475)$. Aquellos pacientes que presentaron alexitimia también presentaron mayor intensidad de fatiga ( $\mathrm{p}=0,0052$ ). Por último, el número de síntomas reportados durante el último mes previo a la evaluación fue significativamente mayor en aquellos pacientes que presentaron alexitimia $(\mathrm{p}=0,0027)$.

Al realizar el análisis multivariado ajustado para las variables que se asumieron como probables factores relacionados a la presencia de alexitimia como: edad, tiempo de enfermedad, presencia de comorbilidades, grado de instrucción y años de estudio, se encontró asociación significativa con años de estudio igual o menor a 11 años ( $\mathrm{p}=0,010$, OR: 2,589 [1,249-5,365]).

\section{DISCUSIÓN}

La principal variable estudiada en el presente estudio fue la alexitimia en relación con diferentes variables tanto sociodemográficas como clínicas. Actualmente no se cuenta con muchos datos nacionales con respecto a dicha variable. En el estudio se encontró una frecuencia de alexitimia de $47,62 \%$ en pacientes con fibromialgia, menor a la reportada en un estudio mexicano donde encontraron una frecuencia de $64,5 \%$. En contraste a la población del presente estudio, en ese grupo se obtuvo una muestra de 102 pacientes, a partir de los 18 años sin límite de edad máxima, además excluyeron a todo paciente de género masculino y pacientes con enfermedades agudas que podían modificar los síntomas (14). Otro estudio que reporta una frecuencia considerable de presencia de alexitimia en pacientes con fibromialgia es el de Elboga y col. (56.4\%), sin embargo, ellos utilizaron la 
Tabla 2. Distribución de acuerdo con la presencia o ausencia de alexitimia

\begin{tabular}{|c|c|c|c|c|}
\hline & $\begin{array}{l}\text { Alexitímico } \\
\text { n (\%) }\end{array}$ & $\begin{array}{l}\text { No alexitímico } \\
\text { n (\%) }\end{array}$ & $\mathbf{p}$ & $\begin{array}{l}\text { OR } \\
\text { (IC:95\%) }\end{array}$ \\
\hline \multicolumn{5}{|l|}{ Sexo } \\
\hline Mujer & $59(98,33)$ & $63(51,64)$ & \multirow{2}{*}{$0,346 *$} & 0,355 \\
\hline Varón & $1(1,67)$ & $3(4,55)$ & & $(0,006-4,608)$ \\
\hline \multicolumn{5}{|l|}{ Comorbilidades } \\
\hline Sí & $43(71,67)$ & $36(54,55)$ & \multirow{2}{*}{$0,047 * * *$} & 2,107 \\
\hline No & $17(28,33)$ & $30(45,45)$ & & $(1,009-4,399) * *$ \\
\hline \multicolumn{5}{|l|}{ Grado de instrucción } \\
\hline Secundaria & $41(68,33)$ & $30(45,45)$ & \multirow{2}{*}{$0,010 * * *$} & \multirow{2}{*}{$2,58(1,177-5,740)$} \\
\hline Superior & $19(31,67)$ & $36(54,55)$ & & \\
\hline \multicolumn{5}{|l|}{ Dolor a la evaluación } \\
\hline Sí & $54(90)$ & $61(92,42)$ & \multirow{2}{*}{$0,63 * * *$} & \multirow{2}{*}{$0,737(0,168-3,091)$} \\
\hline No & $6(10)$ & $5(7,58)$ & & \\
\hline \multicolumn{5}{|l|}{ Depresión } \\
\hline Sí & $24(40)$ & $18(27,27)$ & \multirow{2}{*}{$0,130 * * *$} & \multirow{2}{*}{$1,77(0,788-4,032)$} \\
\hline No & $36(60)$ & $48(72,73)$ & & \\
\hline \multicolumn{5}{|l|}{ Discapacidad } \\
\hline Sí & $39(65)$ & $37(56,06)$ & \multirow{2}{*}{$0,306 * * *$} & \multirow{2}{*}{$1,44(0,667-3,189)$} \\
\hline No & $21(35)$ & $29(43,94)$ & & \\
\hline \multicolumn{5}{|l|}{ Rigidez matutina } \\
\hline Sí & $36(60)$ & $40(60,61)$ & \multirow{2}{*}{$0,94 * * *$} & \multirow{2}{*}{$0,97(0,449-2,119)$} \\
\hline No & $24(40)$ & $26(39,39)$ & & \\
\hline \multicolumn{5}{|l|}{ Ocupación } \\
\hline Empleado & $27(45)$ & $34(51,52)$ & \multirow{2}{*}{$0,465 * * *$} & \multirow{2}{*}{$1,29(0,607-2,779)$} \\
\hline Desempleado & $33(55)$ & $32(48,48)$ & & \\
\hline \multicolumn{5}{|l|}{ Tratamiento farmacológico } \\
\hline Sí & $42(70)$ & $37(56,06)$ & \multirow{2}{*}{$0,106 * * *$} & \multirow[t]{2}{*}{$1,82(0,824-4,091)$} \\
\hline No & $18(30)$ & $29(43,94)$ & & \\
\hline Años de instrucción & & & & \\
\hline Menor o igual a 11 años & $41(68,33)$ & $30(45,45)$ & $0010 * * *$ & $2,589(1,177-5,740)$ \\
\hline Mayor a 11 años & $19(31,67)$ & $36(54,55)$ &, 010 & \\
\hline & Alexitímico & No alexitímico & & \\
\hline & $\mathrm{X} \pm \mathrm{DC}$ & $\mathrm{X} \pm \mathrm{DC}$ & $P$ & \\
\hline Intensidad de dolor & $7,35 \pm 1,83$ & $6,53 \pm 2,17$ & $0,0244 * * * *$ & \\
\hline Edad & $52,15 \pm 11,42$ & $52,39 \pm 9,59$ & $0,823 * * * * *$ & \\
\hline Años de estudio & $11,16 \pm 3,00$ & $12,98 \pm 3,21$ & $0,0016 * * * * *$ & \\
\hline Tiempo de enfermedad (años) & $4,46 \pm 4,76$ & $5,63 \pm 6,94$ & $0,373 * * * * *$ & \\
\hline Número de comorbilidades & $1,26 \pm 1,10$ & $0,89 \pm 0,99$ & $0,0475 * * * * *$ & \\
\hline Tiempo de rigidez (minutos) & $9,16 \pm 15,18$ & $6,60 \pm 12,16$ & $0,52 * * * * *$ & \\
\hline Intensidad de fatiga & $6,98 \pm 2,22$ & $5,75 \pm 2,66$ & $0,0052 * * * * *$ & \\
\hline Intensidad de bienestar & $6,2 \pm 2,51$ & $5,93 \pm 2,05$ & $0,267 * * * * *$ & \\
\hline Número de síntomas reportados & $18,15 \pm 10,50$ & $12,74 \pm 8,21$ & $0,0027 * * * * *$ & \\
\hline Número de fármacos consumidos & $1,18 \pm 0,99$ & $0,90 \pm 1,01$ & $0,086 * * * * *$ & \\
\hline
\end{tabular}


Frecuencia de alexitimia y factores relacionados en pacientes con fibromialgia tratados en un hospital público de Lima.

Tabla 3. Descripción de comorbilidades en la población

\begin{tabular}{lll}
\hline & $\begin{array}{l}\text { Alexitímicos }(\mathbf{n}=\mathbf{6 0}) \\
\mathbf{n}(\mathbf{\%})\end{array}$ & $\begin{array}{l}\text { No Alexitímicos (n=66) } \\
\mathbf{n}(\mathbf{\%})\end{array}$ \\
\hline Artrosis & $13(21,67)$ & $9(13,64)$ \\
Hipertensión arterial & $10(16,67)$ & $9(13,64)$ \\
Diabetes Mellitus tipo 2 & $9(15)$ & $6(9,09)$ \\
Osteoporosis & $7(11,67)$ & $4(6,06)$ \\
Gastritis & $6(10)$ & $3(4,55)$ \\
\hline
\end{tabular}

escala TAS-26 para evaluar la presencia de alexitimia (22). De manera contraria, la frecuencia reportada en nuestro estudio es superior a lo reportado por Castelli y Di Tella, quienes encontraron una frecuencia de alexitimia de $20 \%$ y $25,8 \%$, respectivamente, aunque el número de participantes evaluados en el primer estudio fue mucho menor al nuestro (55 vs. 126), no tuvieron un valor máximo de edad como criterio de exclusión y excluyeron a todo paciente que tenga menos de 5 años de estudio. Sin embargo, en el estudio realizado por Di Tella et al., evaluaron una muestra de 159 pacientes, la cual fue levemente mayor a la nuestra. Los criterios de inclusión y exclusión que usaron fueron similares a los del anterior estudio, exceptuando que también excluyeron aquellos pacientes que presentaron enfermedades neurológicas severas $(16,23)$; por lo que se puede observar, hay estudios que tienen diferencias respecto al nuestro, como las muestras utilizadas, lo que podría explicar en cierto grado los diferentes valores hallados. Sin embargo, hay otros estudios similares metodológicamente al nuestro pero las frecuencias encontradas son muy diferentes, pudiéndose explicar a partir de las características propias de esa población. Otro estudio que presentó una frecuencia de alexitimia considerablemente menor al nuestro fue uno realizado en Chile, donde la frecuencia de alexitimia fue de $10 \%$. Sin embargo, la muestra utilizada fue relativamente pequeña $(\mathrm{N}=30)$ y utilizaron un test no validado en su población para determinar presencia de alexitimia (TAS-20) (12). Por otro lado, cabe mencionar que la frecuencia de alexitimia que encontramos es similar a la hallada en estudiantes de medicina (49\%) en Arabia Saudí (24).

La mayor parte de estudios reportan la media del puntaje del TAS-20, en este estudio la media encontrada fue de 59,4 $\pm 12,2$. Montoro et al., reportan una media similar de 57,44 $\pm 10,94$ (25), Garip y col., señalan una media menor de 41,60土18,63 (15), mientras que Avila et al., hallan una media de 52,0 \pm 13.54 (13). Hay que señalar que estos 3 últimos estudios tuvieron un grupo control, encontrándose que la diferencia entre las medias era estadísticamente significativa, siendo mayor en pacientes del grupo de fibromialgia.

Una variable fuertemente asociada a alexitimia es la presencia de síntomas depresivos, sin embargo, hasta el momento no se ha podido establecer la naturaleza causal entre dichas variables. Por otro lado, se ha reportado mayor relevancia de dicho vínculo dentro de la población con diagnóstico de fibromialgia (26), en contraste a lo anteriormente mencionado, en este estudio si bien se encontró mayor porcentaje de depresión en pacientes con fibromialgia este resultado finalmente no fue significativo $(\mathrm{p}=0,130)$.

Por otro lado, Horta y col. reportaron una relación estadísticamente significativa entre la presencia de alexitimia y las siguientes variables: mayor intensidad de dolor, depresión, percepción de discapacidad y menor calidad de vida. Sin embargo, cuando realizaron el análisis multivariado no hubo resultados estadísticamente significativos para ninguna de las variables mencionadas (14). En nuestro estudio la mayor intensidad de dolor tuvo relación significativa con la presencia de alexitimia en comparación con los pacientes que no tenían alexitimia. En la misma línea, Garip y col. reportan que los pacientes con fibromialgia señalan más dolor en relación a un grupo control $(6,86 \pm 1,58$ vs $1,80 \pm 2,62)(15)$.

En este estudio se halló que tener menos años de instrucción académica se relacionó significativamente con la presencia de alexitimia. Existen estudios epidemiológicos en los que igualmente se encuentra una relación inversa entre el puntaje de la escala de Toronto de Alexitimia y el grado de instrucción $(27,28,29)$. Dentro de los estudios que refuerzan los resultados descritos previamente se encuentra el de Marchi y col. donde se describe una relación negativa con resultados significativos, entre los años de educación y los ítems relacionados a la dificultad de identificar y describir sentimientos dentro de la escala 
TAS-20, relación que se mantiene incluso cuando se controlan las variables de ansiedad y depresión (30). Kauhanen et al., proponen que "por sentido común" se puede asumir que los años de instrucción proveen las "palabras necesarias para los sentimientos". Por otro lado, señalan que considerando la alexitimia como un rasgo de personalidad podría influir negativamente en alcanzar logros académicos en la niñez y adolescencia si se presentan conjuntamente con una alteración cognitiva o que un bajo nivel de alexitimia puede llevar a un mejor desempeño académico (27).

En el Perú, la relación entre la presencia de un síndrome psicopatológico y menos años de instrucción también ha sido reportada por Fudino y col. quienes evaluaron la ansiedad clínicamente significativa en pacientes con artritis reumatoide, encontrándose que los que la presentan tenían menos años de instrucción (31).

Otro de los hallazgos del presente estudio es que los pacientes con alexitimia reportaban mayor número de síntomas en comparación a los pacientes sin alexitimia. Matilla y col. realizaron un estudio poblacional, donde aplicaron los ítems correspondientes a somatización del inventario de síntomas SCL-90 y la escala TAS20. Este grupo encontró que había una relación significativa entre los puntajes de la TAS-20 y los de la sub-escala de somatización del SCL-90 con un coeficiente de correlación de 0,22 ( $\mathrm{p}<0,001)$ (29).

Acerca de la presencia de comorbilidades, en este estudio el $62,7 \%$ presentaban alguna comorbilidad, mientras que Mur y col. indican que el $94 \%$ de pacientes con fibromialgia tenían una comorbilidad asociada. En este mismo estudio, 59,1\% usaban tratamiento farmacológico (7), mientras que en nuestro estudio el porcentaje era similar $(62,7 \%)$.

Existen otros estudios donde además de relacionar la severidad del dolor, capacidad funcional $y$ distrés afectivo en pacientes con presencia de dolor crónico; también incluyen la relación de las variables mencionadas a la presencia de un evento traumático a lo largo de la vida del paciente (32), por lo que se sugeriría realizar estudios similares en nuestra población donde se evalúe la asociación entre fibromialgia, alexitimia y eventos de estrés temprano.

Dentro de las limitaciones, por el diseño del estudio (transversal), no se puede establecer relaciones de causa-efecto. Otra limitación fue que la mayoría de pacientes con diagnóstico de fibromialgia tiene la indicación del uso de amitriptilina, que al ser también un medicamento antidepresivo, podría disminuir la frecuencia de depresión en la población estudiada. Finalmente, si se hubieran incluido pacientes con grado de instrucción primaria probablemente la frecuencia de alexitimia hubiese sido mayor.

En conclusión, en nuestro estudio la frecuencia de alexitimia en pacientes con fibromialgia tuvo un valor considerable (47,62\%). Además, las frecuencias de depresión y discapacidad funcional fueron 33,33\% y $60,32 \%$, respectivamente. No se encontró relación entre la presencia de depresión y alexitimia en los pacientes con fibromialgia. Así mismo, la discapacidad funcional tampoco tuvo relación significativa con la presencia de alexitimia. Dentro de la población alexitímica se encontró significativamente mayor intensidad de dolor. En cuanto a las variables generales la única variable con una relación significativa con la presencia de alexitimia fueron los años de estudio, específicamente con años de estudio menores o iguales a 11.

\section{Correspondencia:}

\section{J. Martín Arévalo Flores \\ Correo electrónico: jorge.arevalo.f@upch.pe}

Conflictos de interés: Los autores declaran que el presente estudio ha sido autofinanciado y niegan cualquier tipo de conflicto de interés.

\section{REFERENCIAS BIBLIOGRÁFICAS}

1. Moyanoa S, Kilsteina J, Alegre C. Nuevos criterios diagnósticos de fibromialgia: ¿vinieron para quedarse? Reumatol Clin. 2015; 11(4):210-214.

2. Alvarado M, Oliva E. Conocimiento, actitudes y resiliencia respecto a su enfermedad en pacientes con fibromialgia de dos hospitales de Lambayeque. Tesis para optar el título de médico cirujano. Chiclayo: Universidad Católica Santo Toribio de Mogrovejo; 2017. 26 pp.

3. Ahmed S, Aggarwal A, Lawrence A. Validation of the proposed 2016 revision to 2010 ACR preliminary fibromyalgia diagnostic criteria in a tertiary care setting. Ann Rheum Dis. 2017; 76:388.

4. Alegre J. La Fibromialgia es una entidad primaria del dolor: ¿Hay deterioro cognitivo en la fibromialgia? Rev Soc Esp Dolor. 2008 ; 15(6): 355-357. (Citado el 5 de marzo del 2020) Disponible en: http://scielo. isciii.es/scielo.php?script=sci_arttext\&pid=S113480462008000600001\&lng=es.

5. Carmona L, Ballina F, Gabriel R, Laffon A. The burden of musculoskeletal diseases in the general 
Frecuencia de alexitimia y factores relacionados en pacientes con fibromialgia tratados en un hospital público de Lima.

population of Spain: results from a nation - wide study. Ann Rheum Dis. 2001; 60:1040-1045.

6. Quevedo H. Incremento en la prevalencia de fibromialgia en un centro médico: un estudio observacional comparando los años 2009 y 2011. Interciencia. 2012; 3(4): 5-9.

7. Mur T, Llordés M, Custal M, López G, Martínez S. Perfil de pacientes con fibromialgia que acuden a los centros de atención primaria en Terrassa. Reumatol Clin. 2017; 13(5): 252-257.

8. Garaigordobil M, Govillard L. Síntomas psicopatológicos en personas con fibromialgia: una reflexión. Interdisciplinaria. 2016; 33(2): 355-374.

9. Galvez C, Reyes G, Duschek S. Cognitive Impairments in fibromyalgia Syndrome: Associations with positive and negative affect, alexithymia, pain, catastrophizing and self-steem. Frontiers in Psychology. 2018; 9:377.

10. Steinweg D, Dallas A, Rea W. Fibromyalgia: unspeakable suffering, a prevalence study of alexithymia. Psychosomatics. 2011; 52(3): 255-262.

11. Ricciardi L, Demartini B, Fotopoulou A, Edwards M. Alexithymia in Neurological Disease: A Review. J Neuropsychiatry Clin Neurosci. 2015; 27(3):179187.

12. Laire X, Rodriguez A, Rodriguez J. Descripcion dimensional de rasgos de personalidad en mujeres con diagnóstico médico de fibromialgia. Revista de Psicología GEPU. 2012; 3(2): 15-35.

13. Avila L, de Araujo G, Guimarães E, Gonçalves L, Paschoalin P, Aleixo F. Characterization of the pain, sleep and alexithymia patterns of patients with fibromyalgia treated in a Brazilian tertiary center. Rev Bras Reumatol. 2014; 54(5): 409-413.

14. Horta G, Peláez I, Queipo G, Montero U, Romero M. Alexithymia is associated with mood disorders, impairment in quality of life and disability in women with fibromyalgia. Clin Exp Rheumatol. 2020; 38 (Suppl 123): 17-24.

15. Garip Y, Öztaş D, Bozkurt Ö, Telci S. Alexithymia in Fibromyalgia Patients and Its Impact on the Quality of Life. J İstanbul Med. 2015; 16: 137-140.

16. Castelli L, Tesio V, Colonna F, Molinaro S, Leombruni $\mathrm{P}$, Bruzzone M, et al. Alexithymia and psychological distress in fibromyalgia: prevalence and relation with quality of life. Clin Exp Rheumato. 2012; 30(74): 7077.

17. Martínez M, Sánchez A, Miró E, Lami J, Prados G, Morales A. Relationships between physical symptoms, emotional distress, and pain appraisal in fibromyalgia: The moderator effect of alexithymia. J Psycho. 2014; 149(2): 115-140.

18. Kendall R, Rodríguez E. Validación de la Escala de Alexitimia de Toronto (TAS-20) en una población de estudiantes universitarios. Tesis para optar el título de especialista en psiquiatría. Lima: Universidad
Nacional Mayor de San Marcos; 2003.

19. Maldonado M. Validación del MD-HAQ (Multidimensional Health Assessment Questionnaire) en un grupo de pacientes con artritis reumatoide de Hospital Nacional del 2 de Mayo. Tesis para optar el título de especialista en reumatología. Lima: Universidad Nacional Mayor de San Marcos; 2005.

20. Ruiz-Grosso P, Loret de Mola C, Vega-Dienstmaier JM, Arevalo JM, Chavez K, Vilela A, et al. Validation of the Spanish Center for Epidemiological Studies Depression and Zung Self-Rating Depression Scales: a comparative validation study. PLoS One. 2012; 7: e45413.

21. Asociación Médica Mundial. Declaración de Helsinki de la AMM - principios éticos para las investigaciones médicas en seres humanos. Washington DC: Asociación médica mundial; 2018. (Citado el 5 de marzo del 2020) Disponible en: https://www.wma. net/es/policies-post/declaracion-de-helsinki-dela-amm-principios-eticos-para-las-investigacionesmedicas-en-seres-humanos/

22. Elboğa G, Serdar M, Altındağ Ö, et al. Alexithymia and attention deficit and their relationship with disease severity in fibromyalgia syndrome. Turk J Phys Med Rehab. 2019; 66(2): 134-139.

23. Di Tella M, Ghiggia A, Tesio V, Romeo A, Colona F, Fusaro E, et al. Pain experience in Fibromyalgia Syndrome: The role of alexithymia and psychological distress. J Affect Disord. 2017; 208: 87-93.

24. Alzahrani H, Coumaravelou S, Mahmoud I, Beshawri J, Algethami M. Prevalence of alexithymia and associated factors among medical students at King Abdulaziz University: a cross-sectional study. Ann Saudi Med. 2020; 40(1):55-62.

25. Montoro I, Reyes G, Duschek S. Alexithymia in fibromyalgia syndrome. Personality and Individual Differences. 2016; 102: 170-179.

26. Ghiggia A, Romeo A, Tesio V, Tella M, Colonna $\mathrm{F}$, Geminiani $\mathrm{G}$, et al. Alexithymia and depression in patients with fibromyalgia: When the whole is greater than the sum of its parts. Psychiatry Res. 2017; 255:195-197.

27. Kauhanen J, Kaplan G, Julkunen J, Wilson T, Salonen J. Social factors in alexithymia. Compr Psychiatry. 1993; 34(5): 330-335.

28. Carril R, Sechrest L, Riedel R. Sociodemographic correlates of alexithymia. Compr Psychiatry. 1998; 39(6): 377-385.

29. Mattila A, Kronholm E, Jula A, Salminen J, Koivisto A, Mielonen R, et al. Alexithymia and somatization in general population. Psychosomatic Medicine. 2008; 70: 716-722.

30. Marchi L, Marzetti F, Orrù G, Lemmetti S, et al. Alexithymia and psychological distress in patients with fibromyalgia and rheumatic disease. Frontiers in Psychology. 2019; 10(1735). 
31. Fudino Y, Salazar J, Wong L, Vega J, Berrocal A. Frecuencia de sintomatología ansiosa y sus factores relacionados en mujeres con artritis reumatoide en un hospital general de Lima. Rev Neuropsiquiatr. 2015; 78 (2): 80-85.
32. Nicol A, Sieberg C, Clauw D, Hassett A, Moser $\mathrm{S}$, Brummett $\mathrm{C}$. The association between a history of lifetime traumatic events and pain severity, physical function, and affective distress in patients with chronic pain. J pain. 2016; 17(12): 1334-1348.

Recibido: 14/07/2020

Aceptado: 29/05/2021 